\title{
PNEUMONIAS EM ADULTOS, ADQUIRIDAS NA COMUNIDADE E NO HOSPITAL
}

\author{
COMMUNITY ACQUIRED AND HOSPITAL - ACQUIRED PNEUMONIAS IN ADULTS
}

Conceição Maria da Costa Santos e Fonseca

Médica Assistente da Divisão de Pneumologia do Departamento de Clínica Médica da Faculdade de Medicina de Ribeirão Preto - USP. Bolsista da Fundação de Apoio ao Ensino, Pesquisa e Assistência do HCFMRP-USP.

CorResPondÊnCIA: Conceição Maria da Costa Santos e Fonseca - Divisão de Pneumologia, Hospital das Clínicas, FMRP-USP CEP 14048-900, Ribeirão Preto, São Paulo, Brasil - Fone: (016) 602-2631 - FAX: (016) 633-6695 - E-mail: cmsantos@usp.br

SANTOS e FONSECA CMC. Pneumonias em adultos, adquiridas na comunidade e no hospital. Medicina, Ribeirão Preto 31: 216-228, abr./jun. 1998.

RESUMO: Neste artigo de revisão, discutem-se fisiopatologia, evolução clínica e aspectos radiográficos de pneumonias comunitárias e hospitalares, em adultos. São abordadas as propostas terapêuticas dos últimos consensos internacionais.

UNITERMOS: Pneumonia. Evolução Clínica. fisiopatologia. radiografia.

\section{PNEUMONIAS EM ADULTOS, ADQUI- RIDAS NA COMUNIDADE}

\section{CONCEITO E EPIDEMIOLOGIA}

Conceitualmente, pneumonia é uma inflamação do trato respiratório inferior. Nesta revisão, abordaremos a pneumonia de origem infecciosa. Trata-se de uma doença bastante freqüente, embora sua real prevalência seja desconhecida, em virtude de não ser de notificação compulsória. Estima-se que ocorram de doze (12) a quarenta (40) casos por mil (1000) habitantes, por ano e, destes, cerca de $80 \%$ são tratados em casa ${ }^{(1)}$.

A maior frequiência de acometimento por pneumonia é observada nos extremos de idade, sendo a distribuição relativamente homogênea, no intervalo de cinco (5) a sessenta (60) anos de idade, em indivíduos previamente hígidos. A mortalidade por pneumonia se relaciona com a faixa etária e com a presença de co-morbidade, sendo maior nos extremos de idade, atingindo cerca de $20 \%$ nos que necessitam internação e $46 \%$ nos idosos com mais de oitenta (80) $\operatorname{anos}^{(2,3)}$.

\section{FISIOPATOLOGIA}

A infecção pulmonar ocorre após um microorganismo ter vencido as barreiras de defesa do hospedeiro (Tabela I). A primeira barreira, a filtração aerodinâmica, é promovida pelas mudanças no regime de fluxo das vias aéreas (turbilhoramento). A segunda barreira é constituída pela mucosa e epitélio da naso e orofaringe. Em conjunto, as duas barreiras favorecem, fazendo com que os microorganismos sejam precipitados, deglutidos, ou eliminados na expiração. Entretanto, se houver aderência do agente ao epitélio, pode haver colonização da via aérea, isto é, a presença do microorganismo no epitélio/mucosa, sem evidências de efeitos adversos ou de reações do hospedeiro, fato que predispõe à infecção. A depuração mucociliar, terceira barreira, promove o aprisionamento do agente no muco e a sua eliminação através da vibração ciliar. Quando a depuração mucociliar é insuficiente para eliminar o agente, desenvolve-se a tosse, que é a quarta barreira. Os componentes celulares (macrófagos e neutrófilos) e os componentes funcionais do ambiente alveolar (imunoglobulinas, complemento e surfactante) constituem a quinta barreira ao agente infeccioso. 
Tabela I - Sistemas de defesa do aparelho respiratório

- Anatomia das vias aéreas: filtração aerodinâmica
- Muco
- Depuração mucociliar
- Reflexo da tosse
- Macrófagos alveolares
- Neutrófilos
- Imunoglobulinas
- Complemento
- Surfactante

A pneumonia pode se desenvolver por diminuição da eficiência dos mecanismos de defesa ou quando a quantidade do agente infectante satura os mecanismos de defesa (Tabela II).

Tabela II - Condições para desenvolvimento da pneumonia

Fonte do agente $\quad$ Vias de contaminação
$\Downarrow$
$\begin{gathered}\text { Pulmão - redução dos mecanismos de } \\ \text { defesa transitório / definitivo } \\ \Downarrow \\ \text { Pneumonia }\end{gathered}$

\section{VIAS DE INFECÇÃO}

Os agentes microbianos podem atingir o parênquima por diferentes vias de acesso ao pulmão (Tabela III). A aspiração de secreções das vias aéreas superiores é a via mais frequiente, e os agentes mais comumente envolvidos são o Diplococcus pneumoniae e o Haemophilus influenzae. A via inalatória permite a instalação de pneumonias por agentes, que ultrapassam a filtração aerodinâmica; são agentes filtráveis,

Tabela III - Vias de acesso do agente ao pulmão

- Aspiração de secreções das vias aéreas superiores

- Inalação

- Hematogênica

- Contigüidade

- Aspiração de conteúdo gástrico

- Translocação

- Inoculação nas vias por procedimentos (ex intubação) como micobactérias, micoplasma sp, fungos e vírus. Através da corrente sanguiínea, via hematogênica, o agente se dissemina para o pulmão, a partir de um foco distante, em qualquer outro tecido; o agente mais freqüente, nessa via de infecção, é o Staphilococcus sp. A infecção pulmonar por via de contigüidade ocorre devido à presença de foco infeccioso em órgão próximo ao pulmão. A infecção, via translocação, ocorre quando há passagem de agentes a partir da luz do tubo digestivo, esta via de infecção pode ser observada em indivíduos com co-morbidades, como hemorragia digestiva, choque ou sepse ${ }^{(4,5,6)}$.

\section{PATOlogia}

A invasão bacteriana do parênquima pulmonar promove solidificação exsudativa do tecido pulmonar (consolidação), caracterizando a pneumonia bacteriana. A apresentação desta forma de pneumonia depende de variáveis, como o agente causal e a reação do hospedeiro, de forma que pode ser classificada de acordo com o agente etiológico (ex.: pneumonia pneumocócica), com a natureza da resposta do hospedeiro (ex.: pneumonia supurativa) ou ainda de acordo com sua distribuição macroscópica (ex.: broncopneumonia ou pneumonia lobar).

A consolidação focal é a característica fundamental da broncopneumonia e, geralmente, representa a extensão de um processo de bronquite ou bronquiolite. A consolidação lobar, pneumonia lobar, constitui-se na infecção bacteriana aguda, que compromete todo um lobo ou grande parte dele. O comprometimento focal (lobular), ou extenso (lobar), do parênquima pulmonar depende das condições de defesa do hospedeiro, da presença de doenças crônicas, deficiências imunológicas, uso de agentes imunossupressores, leucopenias e, mais raramente, da virulência do agente.

Morfologicamente, as pneumonias podem comprometer, predominantemente, a luz alveolar, as vias aéreas e os alvéolos, e o interstício.

Nas formas de comprometimento predominantemente alveolar, na pneumonia lobar, são descritos quatro estágios.

a) Congestão: o lobo apresenta-se pesado, úmido e avermelhado, com ingurgitamento vascular, numerosas bactérias, líquido e neutrófilos na luz alveolar. b) Hepatização vermelha: o lobo apresenta-se avermelhado, firme e desprovido de ar, devido à exsudação maciça e à presença de hemácias, neutrófilos e fibrina na luz alveolar. Esses elementos determinam a consistência endurecida do lobo, de onde provem o termo hepatização vermelha. 
c) Hepatização cinzenta: nesse estágio, ocorre desintegração progressiva das hemácias e persistência do exsudato fibrinossupurativo dentro da luz alveolar, o que dá ao lobo a aparência macroscópica, cinza acastanhada e ressecada.

d) Resolução: estágio final, o exsudado consolidado na luz alveolar sofre digestão enzimática progressiva, e os restos granulares semilíquiidos, reabsorvíveis são eliminados pelos macrófagos ou pela tosse.

Nas formas em que há comprometimento predominantemente das vias aéreas e luz alveolar, broncopneumonia, ocorre inflamação supurativa, que pode ser focal e restrita a um lobo ou, mais freqüentemente, multilobar, bilateral e basal, devido à tendência de as secreções se acumularem nas bases. Os focos atingem diâmetros de $3 \mathrm{a} 4 \mathrm{~cm}$, são de limites pouco precisos, coloração variando de cinza-avermelhada a amarelada, de aspecto granular, seco, e elevam a superfície da área comprometida. Tais focos são ricos em neutrófilos que preenchem brônquios, bronquíolos e espaços alveolares.

Nas formas em que predomina o envolvimento intersticial, pneumonia intersticial, podem ser atingidos lobos inteiros, de um ou ambos os pulmões. As áreas afetadas têm aspecto endurecido, de coloração vermelhoazulada. A reação inflamatória se localiza nas paredes alveolares, os septos alveolares tornamse alargados e infiltrados com células mononucleares (linfócitos, histiócitos e, ocasionalmente, plasmócitos). Em casos agudos, os infiltrados inflamatórios podem conter também neutrófilos. A luz alveolar é livre, entretanto, pode haver comprometimento da luz alveolar por material hemorrágico com restos celulares, fato que reflete a lesão alveolar ${ }^{(7)}$.

\section{ETIOLOGIA}

Inúmeros trabalhos epidemiológicos descrevem as possíveis etiologias para pneumonias adquiridas na comunidade ${ }^{(3,6,8,9)}$. A etiologia das pneumonias é uma variável que sofre influência da faixa etária, da presença de co-morbidades e do grau de comprometimento clínico. O padrão de distribuição dos agentes etiológicos é subdividido em quatro (4) grandes grupos, como mostra a Tabela IV.

\section{CLÍNICA}

A pneumonia, geralmente, se manifesta por tosse, seca ou produtiva, aumento da freqüência respiratória, dispnéia e dor torácica. A febre é manifestação comum, porém pode estar ausente nos indivíduos $\operatorname{idosos}^{(10)}$.

\section{Tabela IV - Etiologia das pneumonias adquiridas na comuni- dade}

Grupo I- Em indivíduo de até sessenta (60) anos de idade sem co-morbidade

- Streptococcus pneumoniae

- Mycoplasma pneumoniae

- Vírus respiratórios (influenzae, para-influenzae)

- Chlamydia pneumoniae

- Haemophilus influenzae

- Legionella sp, Staphylococcus aureus, Mycobacterium tuberculosis

- Fungos (endêmicos); bacilos aeróbios gram-negativos

Grupo II - Em indivíduos com co-morbidade e/ou com mais de sessenta (60) anos de idade

- Streptococcus pneumoniae

- Vírus respiratórios

- Haemophilus influenzae

- Bacilos aeróbios gram-negativos

- Staphylococcus aureus

- Branhamella catarrhalis, Legionella sp. Mycobacterium tuberculosis, Fungos

Grupo III - Em indivíduos que necessitam de hospitalização

- Streptococcus pneumoniae

- Haemophilus influenzae

- Flora mista (incluindo bactérias anaeróbias)

- Bacilos aeróbios gram-negativos

- Staphylococcus aureus

- Chlamydia pneumoniae

- Vírus

- Mycoplasma pneumoniae, Branhamella catarrhalis e fungos

Grupo IV - Em indivíduos hospitalizados com pneumonia grave (que necessitem de CTI)

- Streptococcus pneumoniae

- Legionella sp

- Bacilos aeróbios gram-negativos

- Mycoplasma pneumoniae

- Vírus

- Haemophilus influenzae, Mycobacteria tuberculosis e fungos 
Em geral, quando a evolução da pneumonia é inferior a dez (10) dias, a etiologia bacteriana é a mais provável. Quando a história indica evolução de duração maior do que dez (10) dias, os agentes etiológicos mais prováveis são Micoplasma pneumoniae, vírus, micobactérias e fungos.

A pneumonia promove consolidação pulmonar, que se manifesta, semiologicamente, por diminuição da expansibilidade torácica, aumento do frêmito tóracovocal, macicez à percussão, redução do som vesicular, presença de sopro brônquico (som bronquial), estertores finos, inspiratórios, e aumento da condução da voz (broncofonia/pectorilóquia). Em 20\% dos casos, pode haver sinais clínicos de derrame pleural, ou seja, síndrome de barreira ${ }^{(6,10,11)}$. O diagnóstico de pneumonia é feito com base em critérios principais (anamnese e exame físico) e secundários (exames complementares) (Tabela V). As pneumonias são classificadas, clinicamente, de acordo com o local de aquisição conforme apresentamos na Tabela VI. Na Tabela VII, sugerimos os exames complementares para pacientes que possam ser acompanhados em ambulatórios e para os que necessitam de internação, com o diagnóstico de pneumonia.

\section{Tabela V - Critérios para o diagnóstico de pneu- monia \\ - Critérios principais \\ - Tosse \\ - Expectoração \\ - Febre (Temperatura axilar $\geq 37,8^{\circ} \mathrm{C}$ ) \\ - Critérios secundários \\ - Dor torácica \\ - Dispnéia \\ - Síndrome de condensação \\ - Alteração do estado mental \\ - Exames complementares \\ - Leucometria > 15.000 células $/ \mathrm{mm}^{3}$ \\ - Radiografia de tórax com velamento pulmonar}

\section{EXAMES COMPLEMENTARES NOS PACIENTES COM SUSPEITA DE PNEUMONIA (Tabela VII)}

\section{- Radiografia de tórax}

A radiografia de tórax, em incidência pósteroanterior e lateral deve ser realizada em todo indivíduo com suspeita clínica de pneumonia. Através da radiografia, é possível diferenciar condições que se assemelhem à pneumonia, como as infecções traqueobrônquicas, e evidenciar outras causas, como abscessos pulmonares, lesões cavitárias, obstrução brônquica ou derrames pleurais. A radiografia de tórax também é utilizada, na pneumonia, como critério de gravidade: $\mathrm{o}$ número de segmentos ou lobos comprometidos se correlaciona com a gravidade da doença, ou seja, quanto maior o velamento pulmonar, maior a intensidade do acometimento clínico. Na Tabela VIII, descrevemos o envolvimento pulmonar, a imagem radiográfica, e são sugeridas correlações etiológicas ${ }^{(5)}$. Vale a pena lembrar que não existe aspecto radiográfico que seja exclusivo patognomônico de um único agente, contudo, este é de grande valor, quando associado à clínica.

\section{- Microbiologia da pneumonia}

A detecção do agente causal da pneumonia pode ser feita através de exame das secreções ou do próprio tecido pulmonar (Tabela IX). O exame do escarro é útil na avaliação inicial. A amostra é considerada adequada, se contiver mais de vinte e cinco

\section{Tabela VI - Classificação das pneumonias}

- Pneumonias adquiridas na comunidade

- Forma típica

- Forma atípica

- Forma aspirativa

- Pneumonias em idosos

- De comunidade

- De comunidades fechadas (asilos, casas de repouso, etc.)

- Pneumonias em pacientes imunocomprometidos

- Pneumonias adquiridas no Hospital ou pneumonias nosocomiais

Tabela VII - Exames que devem ser recomendados no quadro de pneumonia

- Para Pacientes de Ambulatório

1. Radiografia de tórax, incidências póstero-anterior (PA) e lateral ( $P$ - perfil)

2. Hemograma completo

- Para pacientes que necessitem de internação

1.Radiografias e Hemograma e mais:

2. Hemocultura

4. Bacterioscopia do escarro direto ou aspirado transtraqueal

5. Broncofibroscopia com lavado brônquico, escovado brônquico (com escova protegida) e biópsia transbrônquica

6. Punção biópsia aspiratória transtoracico

7. Gasometria arterial

8. Bioquímica de função renal: uréia/creatinina 
Tabela VIII - Aspectos radiográficos na pneumonia

O comprometimento pulmonar pode ser: associado ou predominantemente de brônquios, alvéolos e interstícios. Comprometimento:

Apresentação da pneumonia

\section{Alveolar}

Agentes mais freqüentes

- Pneumococcus sp

- M. tuberculosis

- Klebsiella sp

- Legionella sp

Pneumonia intersticial

\section{Intersticial}

Agente mais freqüente:

- Pneumocystis carinii.

Broncopneumonia primária

Broncopneumonia intersticial

Broncopneumonia intersticial supurativa

\section{Acinar / lobular}

Agentes mais freqüentes:

- Pneumococcus sp

De todos os elementos do parênquima

Agentes mais prováveis:

- Mycoplasma pneumoniae,

- vírus (adenovírus, influenza, para

influenza, sincicial respiratório),

- Clamídia pneumoniae

- Coxiella sp

Difuso (vias aéreas, alvéolos e interstícios).

Agentes mais prováveis:

- Staphylococcus

- Bacilos gram-negativos.
- Haemophilus sp.
Aspectos radiológicos

Consolidação homogênea.

Broncograma aéreo.

Distribuição assegmentar.

Ausência de atelectasia.

Pode haver comprometimento pleural.

Consolidação heterogênea lobular ou multilobular, confluente, bilateral e simétrica, sem comprometimento brônquico, de vasos linfáticos e, em geral, sem comprometimento pleural.

Consolidação heterogênea multifocal, acinar (5 a $6 \mathrm{~mm}$ de diâmetro) ou lobular (> 10 mm de diâmetro). Pode haver confluência. Localização em segmentos basais.

Consolidação heterogênea com comprometimento e vias aéreas, alveolos e interstício.

Predomínio na metade inferior dos pulmões.

Consolidação heterogênea.

Distribuição segmentar, confluente ou não.

Ausência de broncograma.

Formação de abscessos.

Insuflação de diminutos abscessos peribrônquicos: pneumatoceles. A ruptura do pneumatocele pode determinar o aparecimento de pneumotórax.
(25) neutrófilos e menos do que dez (10), cinco (5) células epiteliais pavimentosas por campo de baixo poder $^{(2,5,6,8)}$. No escarro, realiza-se exame direto e com colorações, como o Gram, para bactérias inespecíficas, o Ziehl-Nielsen, para bacilos alcoolacidorresistentes (baar), a tinta da China ou a prata para detecção de fungos e outros mais específicos. A cultura do escarro tem baixa especificidade, a menos que sejam utilizados meios específicos, como para baar, Legionella $s p$ e fungos. O emprego de meios de cultura para vírus não é utilizado rotineiramente ${ }^{(1,2,8)}$.

\section{Tabela IX - Técnicas para obtenção de amostras}

- Escarro (espontâneo ou induzido)

- Aspirado transtraqueal

- Broncoscopia:

- Aspiração da secreção brônquica

- Lavado / escovado brônquico

- Lavado broncoalveolar

- Punção biópsia, aspirativa, transtorácica 
Quando a obtenção do espécime é mandatória, técnicas invasivas são utilizadas e consistem de: aspiração transtraqueal, broncoscopia com cateter de coleta protegido, lavado broncoalveolar e o padrão ouro para diagnóstico, a punção biópsia, aspirativa, transtorácica.

A hemocultura deve ser realizada em todo indivíduo que necessite de internação por pneumonia. Devem ser coletadas, pelo menos, duas séries de três $\operatorname{amostras}^{(8,10)}$, colhidas de diferentes locais.

Derrames pleurais devem ser submetidos à toracocentese diagnóstica. Para que o derrame pleural possa ser puncionado, deve haver mais que $500 \mathrm{ml}$ de líquido, na cavidade pleural. A radiografia de tórax, em decúbito lateral do lado comprometido, com raios horizontais, é muito útil, e se evidenciar uma linha de derrame de espessura maior do que $10 \mathrm{~mm}$, sugere que a punção pode ser realizada com segurança. No líquido pleural, determina-se o $\mathrm{pH}$, número de células brancas com contagem diferencial, a concentração de proteínas, glicose e desidrogenase lática (DHL). A proteinemia sérica, a glicemia e a DHL plasmática também devem ser medidas para a verificação da relação entre os respectivos valores no líquido pleural e no plasma. Além disso, deve-se proceder à análise citológica e microbiológica do líquido pleural ${ }^{(11)}$.

Mesmo com o auxílio das técnicas atualmente disponíveis, a identificação do agente etiológico das pneumonias só ocorre em menos de $50 \%$ dos $\operatorname{casos}^{(5,6,10)}$.

\section{ABORDAGEM DO PACIENTE COM SUSPEITA DE PNEUMONIA}

Para abordagem inicial de um indivíduo, acometido com pneumonia adquirida na comunidade, sugere-se o seguinte algoritmo:

a) É pneumonia a doença respiratória desse paciente? História: tosse, dispnéia, dor torácica e febre (critérios principais)

( ) SIM

Hemograma: leucometria $>15.000 \mathrm{~mm}^{3} \quad$ ( ) $\mathrm{SIM}^{*}$

Radiografia tórax: velamento pulmonar ( ) SIM

Três (3) afirmativas $(\mathrm{SIM})=$ Pneumonia.

Para pacientes com idade entre quinze (15) e cinquenta e cinco (55) anos, sorologia para HIV (com consentimento do indivíduo).

\footnotetext{
* Em algumas condições, pode haver leucopenia de valores, inferiores a $4000 / \mathrm{mm}^{3}$, com desvio escalonado.
}

b) Qual a intensidade do comprometimento clínico secundário a pneumonia? ${ }^{(1,6,8,12,13)}$.

Leve $\rightarrow$ Indivíduo sem co-morbidade, em regular estado geral, e com idade inferior a sessenta (60) anos.

Moderada $\rightarrow$ Indivíduo de sessenta (60) anos ou mais, apresentando co-morbidade, com envolvimento radiológico único e sem desaturação arterial.

Grave $\rightarrow$ Indivíduo de qualquer idade, com envolvimento pulmonar múltiplo e/ou extenso, com hipoxemia.

Muito Grave $\rightarrow$ Indivíduo de qualquer idade, com ou sem co-morbidade e com, pelo menos, um dos critérios apresentados na Tabela X.

Tabela X - Critérios para diagnóstico de pneumonia muito grave. A presença de um único critério é diagnóstica

- FR > 35/min

- Hipoxemia grave $\mathrm{PaO}_{2} / \mathrm{FiO}_{2}<250$

- Necessidade de assistência ventilatória mecânica

- Aumento da área de velamento pulmonar na radiografia $\geq 50 \%$ em $48 \mathrm{~h}$

- Choque circulatório (PA sistólico < 90 mm Hg; PAdiastolico $<60 \mathrm{mmHg}$

- Débito urinário $\leq 20 \mathrm{ml} / \mathrm{h}$ ou $\leq 80 \mathrm{ml} / 4 \mathrm{~h}$ (sem doença renal prévia)

- Leucometria $>30.000$ células ou $<4.000$ células / $\mathrm{mm}^{3}$

c) Este paciente pode ser tratado através do ambulatório ou necessita de internação hospitalar? Critérios para sugerir internação:

Idade > sessenta e cinco (65) anos

Frequência respiratória $>35 /$ minuto

PA sistólica $\leq 90 \mathrm{mmHg}$ ou PA diastólica $\leq 60 \mathrm{mmHg}$. Temperatura axilar $\geq 38^{\circ} \mathrm{C}$, mantida por quarenta e oito (48) horas ou mais; ou temperatura axi$\operatorname{lar} \leq 34^{\circ} \mathrm{C}$.

Envolvimento pulmonar múltiplo, ou extenso na radiografia de tórax.

Hemograma: hematócrito $<30 \%$ ou hemoglobina $<9 \mathrm{~g} \%$.

Leucometria: $\geq 30.000$ células $\mathrm{mm}^{3}$ ou $<4.000$ células $\mathrm{mm}^{3}$.

Bioquímica: uréia $>40 \mathrm{mg} \%$ ou creatinina $>1,2 \mathrm{mg} \%$. 
Gasometria arterial $\mathrm{PaO}_{2}<60 \mathrm{mmHg}$ e/ou $\mathrm{PaCO}_{2}$ $\geq 50 \mathrm{mmHg}$, em respiração de ar ambiente.

Existência de doença crônica associada (comorbidade).

Hospitalização no intervalo de um ano, anterior ao quadro atual de pneumonia

Paciente sem condições sociais para tratamento ambulatorial ${ }^{(13)}$.

A presença de três (3) desses critérios sugere que o paciente deva ser internado.

d) Há indicação de terapia intensiva?

A presença de pneumonia grave (5 a $25 \%$ de óbitos) nos primeiros sete dias de internação ou muito grave (50\% de óbitos) constitui indicação para internação em Centro de Terapia Intensiva ${ }^{(14,15)}$.

\section{TRATAMENTO COM ANTIMICROBIANOS}

A terapêutica empírica, para pneumonias adquiridas na comunidade, é orientada segundo a faixa etária, a presença de co-morbidade e o grau do comprometimento clínico ${ }^{(1,2,8,16,17)}$. A duração do tratamento é de dez (10) dias, nas formas leve à moderada, podendo atingir os vinte e um (21) dias, nas formas moderada à grave e muito grave. Nos indivíduos que necessitam de internação, a terapêutica deve ser instituída por via parenteral. Nesses casos, após quatro (4) dias, havendo evidência de melhora clínica e hematológica, podemos fazer a troca da via parenteral para a via oral ${ }^{(8,12,18,19)}$.

Grupo I: indivíduos sem co-morbidade com até sessenta (60) anos de idade, excluídas as imunodeficiências, tratamento ambulatorial taxa de mortalidade: 1 a $5 \%{ }^{(8)}$.

Formas: Leve $\rightarrow$ Moderada

$1^{a}$ sugestão: Macrolídeos: eritromicina, claritromicina, roxitromicina e azitromicina.

$2^{\mathrm{a}}$ sugestão: tetraciclina ou doxiciclina.

$3^{a}$ sugestão: amoxacilina + clavulanato, cefalosporina $2^{\mathrm{a}}$ geração (cefuroxima, cefpodoxime ou cefprozil). Não agem contra micoplasmas, chlamydia e legionella.

Fatores modificantes: suspeita de agente resistente. fluorquinolonas: (levofloxacin, sparfloxacin, ciprofloxacin)

Grupo II: indivíduos com co-morbidade e ou com mais de sessenta (60) anos. Tratamento ambulatorial. Taxa de mortalidade: $5 \%$, porém $20 \%$ podem necessitar de internação durante a evolução.
Formas: Leve $\rightarrow$ Moderada

$1^{\text {a }}$ sugestão: cefalosporina de $2^{\mathrm{a}}$ geração.

$2^{a}$ sugestão: sulfametoxazol + trimetoprima (SMX/TMP).

$3^{a}$ sugestão: betalactâmico + inibidor de $\beta$ lactamase. Podem-se associar macrolídeos.

Grupo III: Indivíduos de qualquer idade com ou sem co-morbidades. Necessidade de Internação (vide critérios para internação). Taxa de mortalidade: 5 a $25 \%$. A maioria dos óbitos ocorreram nos primeiros sete dias de internação.

Formas: Moderada $\rightarrow$ Grave

$1^{\mathrm{a}}$ sugestão: cefalosporina de $2^{\mathrm{a}}$ geração ou de $3^{\text {a }}$ geração (sem atividade antipseudomônica).

$2^{a}$ sugestão: betalactâmico + inibidor de betalactamase. Podem-se associar macrolídeos na suspeita de agente atípico ou rifampicina, se legionella for considerada.

Observação: As cefalosporinas de $3^{\mathrm{a}}$ geração com atividade antipseudomônica podem ser empregadas quando há lesão estrutural pulmonar.

Grupo IV: Indivíduos com pneumonia muito grave. Taxa de mortalidade: $50 \%$.

$1^{a}$ sugestão: macrolídeo + rifampicina, se Legionella for confirmada, associados à: cefalosporina de $3^{\text {a }}$ geração, com atividade antipseudomonas, (está a princípio, somente para pacientes com lesão estrutural pulmonar ou quando for confirmada a presença de pseudomonas) e aminoglicosideo.

$2^{\mathrm{a}}$ sugestão: imipenem - cilastatina

$3^{\text {a }}$ sugestão: Fluorquinolonas: levofloxacina, esparfloxacina, ciprofloxacina.

\section{EVOLUÇÃO}

A pneumonia, quando adequadamente tratada, geralmente, evolui para cura. Entretanto, a presença de fatores de risco alerta o clínico para a possibilidade de desenvolvimento de complicações e infecções metastáticas, que ocorrem em cerca de $10 \%$ dos pacientes com pneumonia pneumocócica e bacteremia. Tais pacientes, podem apresentar meningite, artrite, endocardite, pericardite, peritonite e empiema. Além das infecções metastáticas, podem ocorrer insuficiência renal, insuficiência cardíaca, embolia pulmonar, com ou sem infarto, e até mesmo infarto agudo do miocárdio. A detecção precoce dos fatores de risco (Tabela XI) para o desenvolvimento de complicações, nas pneumonias, é uma forma de evitar a elevação das taxas de mortalidade ${ }^{(13,14,15,20)}$. 
Tabela XI - Fatores de risco para evolução desfavorável na pneumonia

- Idade > 65 anos

- Presença de co-morbidade (cardiopatias, nefropatias, pneumopatia, hepatopatia, diabetes mellitus, doenças cerebrovasculares, esplenectomias, desnutrição, alcoolismo)

- Sinais clínicos: FR > 35/min, FC > 120, PA sistólico $\leq 90$, temperatura axilar $\geq 38^{\circ} \mathrm{C}$ por $\geq 48 \mathrm{~h}$, presença de foco extrapulmonar (infecção metastática), deteriorização do estado de consciência.

- Exames complementares: leucometria $\geq 30.000$ células $/ \mathrm{mm}^{3}$ ou 4.000 células $/ \mathrm{mm}^{3}$ ou neutropenia $<1000$ células $/ \mathrm{mm}^{3}$ )

- Hipoxemia refratária

- Necessidade de assistência ventilatória

- Disfunção renal

- Evidência radiográfica de comprometimento pulmonar múltiplo, presença de modificação radiográfica rápida (< 48 horas) e derrame pleural.

- Anemia (Hematocrito < 30\%; hemoglobina $>9 g \%)$

- Evidência de manifestações por acidose metabólica, aumento do tempo de protrombina, tempo parcial de tromboplastina, redução do número de plaquetas ou produtos de degradação do fibrinogênio $>1: 40$

$\mathrm{FR}=$ Frequencia respiratória; $\mathrm{FC}=$ Frequencia cardiaca

\section{PNEUMONIAS DE EVOLUÇÃO ATÍPICA}

A pneumonia é uma doença aguda e, em geral, está resolvida dentro do intervalo de quinze (15) dias de tratamento, em média. Entretanto, existem formas de comportamento atípico ${ }^{(21,22)}$, que costumam provocar erros no diagnóstico e, algumas vezes, podem determinar a realização de procedimentos invasivos precocemente. As pneumonias de evolução atípica são conceitualmente classificadas de acordo com a sua evolução temporal:

- Pneumonia de resolução lenta

- Pneumonia crônica

- Pneumonia recorrente

\subsection{Pneumonia de resolução lenta}

É a pneumonia adquirida na comunidade por indivíduo imunocompetente, em que há melhora clínica, e apenas 50\% de "limpeza" radiográfica, após duas (2) semanas de evolução com terapêutica adequada. A resolução radiográfica ocorre após quatro (4) semanas. Em conclusão, trata-se de resolução radiológica lenta. É encontrada em indivíduos idosos e em portadores de co-morbidades como insuficiência cardíaca ou renal.

\subsection{Pneumonia recorrente}

É aquela que acomete o indivíduo com frequiência maior do que duas (2) vezes por ano, com intervalo maior do que trinta (30) dias entre os episódios, na ausência de diagnóstico de micobacteriose, doença fúngica ou neoplásica. A doença mais comumente associada à pneumonia recorrente é a DPOC, segui$\mathrm{da}$, em ordem de frequiência, por bronquiectasias, fibrose cística, síndrome de cílio imóvel e sequiestro pulmonar. Das doenças extratorácicas, as que mais se correlacionam com pneumonia recorrente são alcoolismo (agudo e crônico) e a diabetes mellitus.

\subsection{Pneumonia crônica}

É aquela que acomete indivíduo imunocompente e que evolui com evidências clínicas e radiográficas de doença em atividade por mais de um (1) mês, na ausência de tumores, bronquiectasias, bronquite crônica, corpo estranho nas vias aéreas ou imunodeficiência. Após dois (2) meses, em geral, ocorre resolução do quadro e o indivíduo evolui de forma assintomática. A investigação de outra doença está autorizada após oito (8) semanas de acompanhamento e, só então, as técnicas invasivas para diagnóstico etiológico da lesão pulmonar devem ser empregadas. Contudo, confirmada a patologia de pneumonia, esse paciente deve ser acompanhado por, pelo menos, seis (6) meses, período em que não haverá diagnóstico adicional, o que confirmaria a hipótese de pneumonia crônica. As doenças que estão mais freqüentemente associadas ao desenvolvimento da pneumonia crônica são: DPOC, cardiopatia, nefropatia, diabetes mellitus, alcoolismo, acidente vascular encefálico e refluxo gastroesofágico $^{(21)}$.

\section{PNEUMONIAS ATÍPICAS}

Originalmente, o termo foi usado para descrever quadros de pneumonia adquirida na comunidade, cujo comportamento diferia do padrão comumente observado. Em comum, nessas pneumonias, não se conseguia detectar o agente pelos meios de cultura 
habitualmente empregados, a intensidade do acometimento clínico variava de leve a moderado e se arrastava por até quatro semanas, com persistência do quadro de tosse, em geral, seca e, por vezes, produtiva, febre, geralmente sem sintomas pleurais, e, notadamente, ausência de resposta a agentes betalactâmi$\cos ^{(22)}$. Finalmente, um agente foi isolado: Mycoplasma pneumoniae e, portanto, pneumonia atípica tornou-se sinônimo de pneumonia por Mycoplasma pneumoniae. Posteriormente, quadros de pneumonias atípicas foram descritos com outras espécies, como a Legionella sp ou Clamídia pneumoniae cepa Twar $\mathrm{e}$, historicamente, o termo pneumonia atípica passou a ser referência para infecção pulmonar provocada por um desses agentes. Contudo, o termo vem sendo utilizado, também, para descrever quadros de pneumonia cuja evolução se desvie do padrão comum e que não são necessariamente provocados por esses agentes $^{(21,22)}$.

\section{DURAÇÃO DO TRATAMENTO}

A duração da antibioticoterapia depende da presença de co-morbidades, de bacteremia, da gravidade do acometimento provocado pela pneumonia e da evolução clínica. Em geral, pneumonias bacterianas devem ser tratadas por dez (10) dias em média. Formas atípicas devem ser tratadas por quinze (15) dias em média ${ }^{(2,8,14)}$.

A troca da via parenteral para a via oral para administração da antibioticoterapia depende de fatores do paciente (se já pode deglutir, e se a absorção intestinal está preservada) e do antimicrobiano (equipotência parenteraloral ou não haver mais necessidade de níveis elevados. Contudo, após esterilização clínica, com melhora e ausência de febre por mais de $24 \mathrm{~h}$, a troca pode ser feita do terceiro ao sexto dia de antibioticoterapia $^{(8)}$.

\section{PNEUMONIAS EM ADUlTOS, ADQUI- RIDAS NO HOSPITAL}

\section{CONCEITO E EPIDEMIOLOGIA}

As pneumonias adquiridas no ambiente hospitalar representam a segunda principal causa de infecção hospitalar nos Estados Unidos, e estão associadas a altas taxas de morbidade e mortalidade ${ }^{(4,9,23)}$. E o que é pneumonia hospitalar? Por conceito, trata-se de infecção do trato respiratório inferior, que ocorre após setenta e duas (72) horas da internação hospitalar, em indivíduo previamente livre de infecção respiratória, ou da súbita piora clínica e radiográfica em um quadro de pneumonia que evoluía favoravelmente ${ }^{(23)}$.

A mortalidade na pneumonia hospitalar é estimada em $70 \%$ dos casos, contudo, essa mortalidade, dependendo do agente, pode ser maior, como ocorre nos casos de pneumonia hospitalar por Pseudomonas aeruginosa ou Acinetobacter sp ${ }^{(9)}$.

A pneumonia hospitalar é classificada, evolutivamente, em precoce e tardia. Tal divisão tem o objetivo de identificar os agentes etiológicos de acordo com o local aquisição. Pneumonias hospitalares, diagnosticadas nos cinco (5) primeiros dias após a internação, são classificadas como precoces, e os agentes são, em geral, os mesmos da pneumonia adquirida na comunidade. Após cinco (5) dias de internação, a pneumonia hospitalar é classificada como tardia e os agentes são, com maior frequiência, de origem hospitalar.

A pneumonia hospitalar é pouco diagnosticada, por ser confundida com outras doenças e porque pode se manifestar somente após a alta hospitalar ${ }^{(9)}$.

\section{ETIOLOGIA}

Os agentes etiológicos nas pneumonias hospitalares podem diferir nos diversos ambientes e embora agentes bacterianos sejam os mais freqüentemente isolados, outros possíveis agentes como vírus, Legionella sp., anaeróbios e fungos, não são pesquisados de rotina.

As fontes de infecção se encontram no ambiente (hospital, os aparelhos), em outros pacientes e na equipe de funcionários (médicos, enfermeiros, estudantes). Na Tabela XII, apresentamos os agentes etiológicos mais freqüentes, de acordo com a possível fonte ${ }^{(9,23)}$. Na Tabela XIII, apresentamos os fatores de risco associados ao desenvolvimento de pneumonias hospitalares.

Na suspeita da pneumonia hospitalar, devemos proceder à investigação de forma semelhante à de pneumonia comunitária, com radiografia de tórax nas incidências póstero-anterior e perfil, hemograma, hemocultura e coleta de material pulmonar (escarro espontâneo ou induzido, aspirado transtraqueal ou brônquico, lavado brônquico e biópsia). Na suspeita de pneumonia hospitalar, essa bateria de exames para a confirmação diagnóstica deve ser prontamente realizada, pois a demora predispõe a evolução desfavorável. 
Tabela XII - Fontes de infecção na pneumonia adquirida no hospital e possíveis agentes

1) Ambiente

- $\mathrm{Ar}=$ Aspergillus sp, Staphylococcus aureus, vírus sincicial respiratório

- Água = Legionella $s p$

- Alimentos = Bacilos entéricos gram-negativos

- Aerossóis $=M$. tuberculosis

2) Aparelhos

- Tubos endotraqueais

- Cateteres de aspiração

- Broncoscópios

- Equipamentos de fisioterapia respiratória

- Sondas nasogástricas

3) Outros Pacientes

- Vírus: influenzae, Sincicial respiratório

- Haemophilus influenzae

- Staphilococcus aureus (meticilinorresistentes)

- Pseudomonas aeruginosa

4) Corpo Clínico

- Vírus: Influenzae, Sincicial respiratório

- Haemophilus influenzae

- Staphylococcus aureus (cepas resistentes)

- Pseudomonas aeruginosa

Tabela XIII - Fatores de risco para desenvolvimento da pneumonia hospitalar

- Fatores do hospedeiro

- Idade $\geq$ sessenta (60) anos,

- Co-morbidade, DPOC/insuficiência respiratória/ alcoolismo/rinosinusopatias, nefropatias, cardiopatias, sinusopatia)

- Distúrbios da função consciente.

- Uso prévio de antibiótico(s)/corticosteroides/quimioterápicos

- Uso de bloqueadores $\mathrm{H}_{2}$ /antiácidos

- Colonização gástrica

- Choque

- Cirurgias recentes (notadamente as abdominais)

- Assistência ventilatória por mais de quarenta e oito (48) horas

\section{CLASSIFICAÇÃO CLÍNICA}

A pneumonia adquirida no hospital é também classificada em leve, moderada, grave, muito grave e subdividida em grupos, de acordo com a presença de fatores de risco, o período de instalação, se precoce ou tardio, o comprometimento físico do paciente e a causa da internação, notadamente internação por motivos cirúrgicos e o seu porte ${ }^{(8,12,13,23,24)}$.

Grupo I: São alocados os casos de pneumonia hospitalar classificada de leve a moderada, em indivíduos sem fatores de risco específicos. A instalação da pneumonia pode ser precoce ou tardia. Também fazem parte deste grupo os casos de pneumonia hospitalar muito grave, de instalação precoce. Os fatores de risco são inespecíficos, entretanto, o tempo de internação e a idade do indivíduo contribuem para o desenvolvimento do quadro. Os agentes de maior prevalência são: bacilos anaeróbios, enterobactérias (Escherichia coli, Klebsiella sp, Proteus sp, Serratia marcescens, Enterobacter), Haemophilus influenza, Staphylococcus aureus (meticilinorresistente), Streptococcus pneumoniae, Pseudomonas aeruginosa, Acinetobacter.

Grupo II: Estão classificados os casos de pneumonia hospitalar classificada de leve a moderada, em indivíduos com fatores de risco específicos, independente do período da instalação (precoce ou tardia). Os agentes de maior prevalência são os anaeróbios, quando os fatores de risco são cirurgia abdominal recente ou aspiração do conteúdo gástrico. O Staphylococcus aureus é o agente etiológico, quando os fatores de risco são coma, traumatismo craniano, diabetes mellitus e insuficiência renal. Quando o fator de risco é o uso de imunossupressores: Legionella $s p$. Quando os fatores de risco são doença estrutural do pulmão (exemplo: bronquiectasia, cistos), uso de imunossupressores, antibióticos por tempo prolongado e longas internações nas unidades de terapia intensiva: Pseudomonas aeruginosa.

Grupo III: Estão alocados os casos de pneumonia hospitalar muito grave, de instalação precoce em indivíduos nos quais os fatores de risco são lesões estruturais do pulmão, uso de antibióticos, imunossupressores e longos períodos de assistência ventilatória. Também estão nesse grupo os casos de pneumonia hospitalar grave de instalação tardia. Os agentes de maior frequiência são a Pseudomonas aeruginosa, o Acinetobacter e o Staphylococcus aureus meticilinorresistente. 
Quando se confirma o diagnóstico de pneumonia hospitalar, a decisão terapêutica deve ser guiada pela classificação dos grupos, conforme mostrado na Tabela XIV. A terapia inicial pode ser modificada, dependendo dos resultados dos exames complementares, como a bacterioscopia da secreção pulmonar, das hemoculturas e cultura das secreções, ou, ainda, após tempo mínimo de quatro (4) dias, com evidente piora clínica, descartadas outras possibilidades para descompensação do indivíduo ${ }^{(9,20,23,24,25)}$.

\section{COMENTÁRIOS}

A pneumonia é um problema de saúde pública. De alta incidência, principalmente nos meses de inverno, ocorre durante todo o ano e detém alta morbidade e mortalidade. Sua importância epidemiológica tem aumentado progressivamente. Esse fato se correlaciona com o aumento da longevidade, o emprego de técnicas terapêuticas imunossupressoras e a epidemia de infecção pelo vírus HIV.

Apesar de as diferentes formas de apresentação das pneumonias serem amplamente reconhecidas, a distinção entre pneumonia adquirida na comunidade, pneumonia hospitalar e as síndromes de pneumonia atípicas, baseada em critérios clínicos, radiológicos e mesmo microbiológicos, é imprecisa, pois ainda não existem critérios de significativa especificidade. Portanto, a história da moléstia atual é de grande auxílio na interpretação e valorização dos resultados obtidos.

Quanto à abordagem terapêutica, o consenso da "American Thoracic Society", de 1993, sugere a classificação da pneumonia adquirida na comunidade em grupos e a partir daí o tratamento. Esse consenso sugere a mudança na abordagem terapêutica da pneu- monia $^{(8)}$. Considerando-se o tempo de publicação do consenso, atualmente, é possível fazer discussões, incluindo as discordâncias a seu respeito $(13,17,18,19,26)$. A revisão dessa literatura médica, em conclusão, sugere que a terapêutica proposta possa não ser tão eficiente quanto se espera, exceto no Grupo I, onde parece haver uma tendência ao acordo. Nesses indivíduos do Grupo I (idade inferior a sessenta e cinco (65) anos, sem co-morbidades, e estadiamento clínico leve a moderado), o tratamento das pneumonias adquiridas na comunidade com eritromicina foi o de mais baixo custo e o de melhor resposta ${ }^{(19)}$. O grande inconveniente do seu uso, o efeito irritante do tubo digestivo, pode, atualmente, ser contornado pelo uso de outro macrolídeo de segunda geração (azitromicina, claritromicina ou roxitromicina).

Para os outros grupos, o tratamento ainda poderia seguir as orientações anteriores, uma vez que o sugerido pelo consenso aumenta o custo, sem reduzir a mortalidade ${ }^{(19,26)}$. Tradicionalmente, o esquema para tratamento empírico das pneumonias adquiridas na comunidade em indivíduos imunocompetentes utiliza antibióticos $\beta$ lactâmicos. Na primeira abordagem, isso ocorre porque, na grande maioria dos levantamentos epidemiológicos, o Diplococcus pneumoniae é responsável por mais de $60 \%$ dos $\operatorname{casos}{ }^{(1,2,3,8,13,14,16,17,19)}$, e a diminuição da sensibilidade ou resistência do microorganismo ao agente só começou a surgir nos últimos anos.

Com tal consideração, a Sociedade Americana de Doenças Infecciosas ${ }^{(13)}$ sugere, em seu consenso de 1998, que se tente a detecção do agente infeccioso, realizem-se testes de sensibilidade, que sejam utilizados os $\beta$ lactâmicos segundo a concentração inibitória mínima. Quando a concentração inibitória mínima for menor que $0,1 \mu \mathrm{g} / \mathrm{ml}(\mathrm{CIM}<0,1 \mu \mathrm{g} / \mathrm{ml})$, a cepa

\begin{tabular}{|c|c|c|}
\hline Grupo I & Grupo II & Grupo III \\
\hline $\begin{array}{l}\text { - Cefalosporina } 2^{a} \text { geração (g) } \\
\text { ou } 3^{a} \mathrm{~g} \text { sem ação antipseudo- } \\
\text { monas ou Betalactâmicos + ini- } \\
\text { bidor da betalactamase } \\
\text { - Para alérgicos à penicilina: } \\
\text { fluorquinolona ou clindamicina + } \\
\text { aztreonam. }\end{array}$ & $\begin{array}{l}\text { - Clindamicina ou betalactâmi- } \\
\text { cos + inibidor de betalactamase } \\
\text { associado } \\
\text { Pode-se usar: } \\
\text { (com ou sem) Vancomicina ou } \\
\text { teicoplamina (até que seja des- } \\
\text { cartada presença de estafilo- } \\
\text { coco resistente). } \\
\text { - Eritromicina ou Rifampicina. } \\
\text { Pode ser usada associada na } \\
\text { suspeita forte de Legionella }\end{array}$ & $\begin{array}{l}\text { - Aminoglicosídeos ou flu- } \\
\text { orquinolonas acrescido de } \\
\text { um dos seguintes antimi- } \\
\text { crobianos: betalactâmico + } \\
\text { inibidor de betalactamase; } \\
\text { imipenem-cilastatina cefta- } \\
\text { zidima ou cefoperazone; } \\
\text { aztreonam (no caso de ba- } \\
\text { cilo entérico gram negati- } \\
\text { vos); com ou sem vanco- } \\
\text { micina ou teicoplamina. }\end{array}$ \\
\hline
\end{tabular}


apresenta alta sensibilidade à droga e a de escolha deverá ser a penicilina $\mathrm{G}$ ou amoxicilina. Para cepas de sensibilidade intermediária (CIM $>0,1 /<1 \mu \mathrm{g} / \mathrm{ml}$ ), penicilina $\mathrm{G}$ ou amoxicilina associada a drogas de comprovada resistência às $\beta$ lactamases (ácido clavulânico/ticarcilina). Para cepas resistentes (CIM $\geq 2 \mu \mathrm{g} / \mathrm{ml}$ ), vancomicina, fluoroquinolona ou outros agentes de sensibilidade comprovada.

O tratamento de pneumonia hospitalar, proposto pela ATS ${ }^{(9)}$, necessita ser validado para que se possa realmente avaliar a sua eficácia ${ }^{(9,20,23,25)}$.
Em nosso meio, como em outros centros, o tratamento de pneumonias comunitárias e hospitalares também tem sido norteado pelos consensos, embora muitos médicos ainda prefiram esquemas terapêuticos tradicionais ${ }^{(3,16,23,25)}$.

AGRADECIMENTOS: Aos Professores Doutores João Carlos da Costa (Divisão de Moléstias Infecciosas) e José Carlos Manço (Divisão de Pneumologia), pela revisão deste artigo, e às senhoras Rosa Maria Pereira Coquely e Ednéia Fabbris Verceze, pela digitação.

SANTOS e FONSECA CMC. Community acquired and hospital - acquired pneumonias in adults. Medicina, Ribeirão Preto 31: 216-228, apr./june 1998.

ABSTRACT: In this review, we discuss pathophysiology, clinical and radiological aspects of community - acquired and hospital - acquired pneumonias. Therapeutic trials are presented as proposed by international consenses.

UNITERMS: Pneumonia. Clinical Evolution. Physiopathology. radiography.

\section{REFERÊNCIAS BIBLIOGRÁFICAS}

1 - BARTLETT JG \& MUNDY LH. Community-acquired pneumonia: currents concepts. N Engl J Med 33: 1618-1623, 1995.

2 - MANDELL LA. Community acquired pneumonia. Etiology, epidemiology and treatment. Chest 108: 35S-42S, 1995.

3 - WOODHEAD MA et al. Prospective study of the aetiology and outcome of pneumonia in the community. Lancet 1 : 671-674, 1987.

4 - VALERY MIBA. Pneumonia adquirida no hospital. In: CUKIER A, NAKATANI J \& MORRONE N, eds. Pneumologia: Atualização e reciclagem, 2 ed. Editora Atheneu, São Paulo, cap. 32, p. 208-214, 1997.

5 - CORREA DA SILVALC et al. Pneumonias. In: CORREA E SILVA LC, ed. Compêndio de pneumologia, Editora BycProcienx, São Paulo, cap. 22, p. 245-60, 1995.

6 - HOPKINS CC. Community acquired pneumonia. In: FISHMAN AP, ed. Pulmonary disease and disorders. 2 th ed. McGraw-Hill Book, New York, p. 1535-1541, 1985.

7 - KOBZIK L; SCHOEN FJ. O pulmão. In: COTRAN RS; KUMAR V; SCHOEN FJ \& ROBBINS SL, ed. Patologia estrutural e funcional. $5^{\text {a }}$ ed. Editora Guanabara Koogan, Rio de Janeiro, cap. 15, p. 598-654, 1994.

8 - AMERICAN THORACIC SOCIETY, MEDICAL SECTION OF AMERICAN LUNG ASSOCIATION: Guidelines for initial management of adults with community-acquired, pneumonia: diagnosis, assessment of severity and initial antimicrobial therapy. Am Rev Respir Dis 148: 1418-1426, 1993.
9 - AMERICAN THORACIC SOCIETY, Medical Section of American Lung Association. Hospital - Acquired Pneumonia in Adults: Diagnosis, assessment of severity, initial antimicrobial therapy, and preventive strategies. A consensus statement. Am J Respir Crit Care Med 153: 1711-1725, 1996.

10 - SWARTZ MN. Aproach to the patient with pulmonary infections. In: FISCHMAN AP, ed. Pulmonary disease and disorders. 2 th ed. McGraw-Hill Book, New York, p. 13751410, 1985.

11 - SAHN SA. State of art: The pleura. Am Rev Respir Dis 138: 184-234, 1988.

12 - FINE MJ et al. The hospital discharge decision for patients with community acquired pneumonia. Results from the pneumonia patients outcomes research team cohort study. Arch Intern Med 157: 47-56, 1997.

13 - BARTLETT JG et al. Guideline from the infectious disease society of América community - acquired pneumonia in adults: guideline for management. Clin Infect Dis 26: 811-838, 1998.

14 - BRITISH THORACIC SOCIETY RESEARCH COMMITTEE AND PUBLIC HEALTH LABORATORY SERVICE. The aetiology, management and outcome of severe community-acquired pneumonia in the intensive care unit. Respir Med 86: 7-13, 1992.

15 - ALMIRALL $\mathrm{J}$ et al. Prognostic factor of pneumonia requiring admission to the intensive care unit. Chest 107: 511-516, 1995.

16 - GREEN M \& WALD ER. Emerging resistance to antibiotics: Impact on respiratory infections in the outpatient setting. Ann Allergy, Asthma \& Immunol 77: 167-173, 1996. 
17 - MANDELL LA \& NIEDERMAN HS. The canadian community acquired pneumoniae consensus group. Antimicrobial treatment of community acquired pneumonia in adults: a conference report. Can J Infect Dis 4: 25-28, 1993.

18 - FEIN AM \& NIEDERMAN MS. Guidelines for initial management of community-acquired pneumonia: Savory Recipe or Cookbook for Disaster, clinical commentaries. Am J Respir Crit Care Med 152: 1149-1153, 1995.

19 - GLEASON PP et al. Medical outcomes and antimicrobial costs with the use of the American Thoracic Society Guidelines for outpatients with community acquired pneumonia. JAMA 278: 32-39, 1997.

20 - TABLON OC et al. Guideline for prevention of nosocomial pneumonia. P.I.: Issues on prevention of nosocomial pneumonia, 1994. Infect Control Hosp Epidemiol 15: 588-625, 1994.

21 - KIRTLAND S \& WINTIRBAUM R. Slowly resolving, chronic and recurrent pneumonia. Atypical Pneumonia syndromes. Clin Chest Med 12: 303-318, 1991.
22 - WINTERBAUER RH. A typical pneumonia syndromes. Clin Chest Med 12: 203-407, 1991.

23 - CRAVEN DE, STEJER KA. Epidemiology of nosocomial pneumonia: new perspectives on an old disease. Chest 108: 1S-16S, 1995.

24 - CRAVEN DE; STEFER KA \& BARBER TW. Preventing nosocomial pneumonia: State of art and perspectives for 1990's. Am J Med 91(3B) S44-S53, 1991.

25 - BERGOGNE-BÉRÉZIN E. Therapy and prevention of nosocomial pneumonia. Chest 108: 26S-34S, 1995.

26 - HIRANI NA \& MACFARLANE JT. Impact of management guidelines on the outcome of severe community acquired pneumonia. Thorax 52: 17-21, 1997.

Recebido para publicação em 13/05/98

Aprovado para publicação em 10/06/98 